\title{
Factors associated with fatigue in patients with systemic lupus erythematosus
}

\author{
Ian N Bruce, Vincent C Mak, David C Hallett, Dafna D Gladman, Murray B Urowitz
}

\begin{abstract}
Objective-To examine the relation between fatigue, disease activity, damage, and quality of life measures in patients with systemic lupus erythematosus (SLE). Methods-Consecutive patients attending the University of Toronto Lupus Clinic were studied. Disease activity was assessed using the SLEDAI and SLAM-R and damage using the SLICC/ACR Damage index. Fatigue was measured by the Fatigue Severity Score (FSS) and health status by the SF-36 questionnaire. In all cases a tender point count was also performed.

Results-81 patients were studied. Their mean (SD) age and disease duration were $43(12.5)$ years and 12.7 (8.0) years respectively. The FSS did not correlate with the SLEDAI nor with the SLAM-R. There was no correlation with the SLICC damage index. Fatigue severity correlated with the tender point count (SCC $r=0.46, p<0.001$ ), and negatively with all domains of the SF36 ( $r$ values -0.50 to -0.82 ). Disease activity and damage accounted for only $4.8 \%$ and $4 \%$ respectively of the variance in fatigue severity reported by patients.

Conclusion-In an outpatient population of SLE patients, fatigue severity correlates with poor health status and a higher tender point count. In patients with SLE, factors associated with quality of life and fibromyalgia seem to have a greater influence on the severity of reported fatigue than does the level of current disease activity.

(Ann Rheum Dis 1999;58:379-381)
\end{abstract}

Fatigue is a very common symptom in systemic lupus erythematosus (SLE) and is described in over $50 \%$ of patients at some time during their illness. ${ }^{12}$ The cause of fatigue seems to be multifactorial although it is often interpreted as reflecting active disease. As such, it is included in several indices of disease activity. ${ }^{3}$ However, other conditions associated with fatigue also occur frequently in SLE including fibromyalgia, which has been reported in up to $22 \%$ of patients. ${ }^{4} 5$

In a previous study, ${ }^{6}$ we found that disease activity as assessed by the SLEDAI did not correlate with patients' reporting of fatigue. This study suggested that fatigue was more closely associated with quality of life (QOL) (measured by the Medical Outcome Survey (MOS) SF-20), depression, and fibromyalgia. The potential contribution of accumulated organ damage to fatigue was not assessed.

The purpose of this study was to further examine which dimensions of disease in SLE are most closely associated with fatigue. We studied the relation of fatigue with disease activity using two indices, one that includes $\left(\right.$ SLAM-R) ${ }^{3}$ and one that excludes (SLEDAI) ${ }^{7}$ fatigue. We also assessed the relation of fatigue with overall damage, tender point count, and health related QOL, using the MOS SF-36, which is the accepted QOL measure for studies of SLE. ${ }^{8}$

\section{Methods}

PATIENT SELECTION

Consecutive patients attending the University of Toronto Lupus Clinic between March and May 1997 were included. All patients fulfilled ACR criteria for the Classficiation of SLE. ${ }^{9}$

\section{CLINICAL EVALUATION}

Patients with SLE are followed up prospectively at the University of Toronto Lupus Clinic. Our standard protocol includes all features of active disease and damage encountered by patients with SLE. It also includes a question on fatigue, as well as the assessment of fibromyalgia and a fibromyalgia tender point count at each visit. The SLEDAI ${ }^{7}$ is then calculated from the information collected on the protocol, and every 12 months the SLICC/ ACR damage index is completed..$^{10}$ For patients who had not had a SLICC/ACR damage index within six months of the current visit, it was completed again for the current visit. All patients are assessed by the directors of the clinic (Dr M B Urowitz or Dr D D Gladman) or by a clinical fellow trained by them.

ADDITIONAL ASSESSMENTS OF ACTIVITY, QUALITY OF LIFE, AND FATIGUE

During the patient's visit in this time period, the following additional measures were completed:

The SLAM-R was completed for each patient. The SLAM includes a severity measure for some of the items, as well as some items, 
Table 1 Demographic details for each scale of activity, damage, fatigue and quality of life assessed in the patient population. The normal data for each domain in the SF-36 for the US population are displayed for comparative purposes

\begin{tabular}{lll}
\hline Measures & $\begin{array}{l}\text { SLE patients } \\
\text { (mean (SD) })\end{array}$ \\
\hline Total number studied & 81 \\
Women/men & $76 / 5$ & \\
SLEDAI & $5.4(5.0)$ & \\
SLAM-R & $6.33(3.9)$ & \\
SLICC & $1.49(1.6)$ & \\
Fatigue severity score & $6.08(2.7)$ & \\
SF-36 domains & & \\
Physical function & $63.2(32.4)$ & $81.5(24.6)$ \\
Role physical & $52.5(46.1)$ & $77.8(36.2)$ \\
General health & $48.7(24.6)$ & $70.6(21.5)$ \\
Bodily pain & $58.9(26.7)$ & $73.6(24.3)$ \\
Vitality & $47.0(24.4)$ & $58.4(21.5)$ \\
Social function & $66.1(29.0)$ & $81.5(23.7)$ \\
Role emotional & $66.0(43.5)$ & $79.5(34.6)$ \\
Mental health & $67.8(21.6)$ & $73.3(18.7)$ \\
&
\end{tabular}

including fatigue, arthralgia and myalgia, which are not part of SLEDAI. This information was included in the standard assessment such that the SLAM could be completed as well. The clinical fellows involved in the study (INB and VCM) were instructed in the guidelines for use of this instrument, including the rules regarding attribution.

The patients also completed the following instruments:

(1) Fatigue Severity Score (FSS), this is a validated nine point questionnaire that assesses the effect of fatigue on daily activities. ${ }^{11}$ Each item is scored on a scale from $0-10$, as modified from the original 1-7 scale by Gladman et $a l^{12}$ with an average overall score (0-10) being computed; a higher score denotes more severe fatigue.

(2) The Medical Outcomes Study 36-item short form health survey (SF-36). This is an extensively used measure of health related QOL. ${ }^{13}$ The questionnaire covers eight domains of health status - that is, physical function, role physical, role emotional, social functioning, bodily pain, mental health, vitality, and general health. Scores range from 0-100 in each domain with a higher score reflecting better performance.

Patients completed the SF-36 and FSS independent of their visit with the physician. The questionnnaires were returned to the research assistant and entered on computer. The physicians were blinded to the results of the patient derived questionnaires.

STATISTICAL ANALYSIS

Statistical analysis was performed using the SAS for PC package. The mean (SD) for each variable were calculated. Correlation analyses were calculated using Spearman's coefficient of correlation. Ninety five per cent confidence intervals $(95 \% \mathrm{CI})$ and $\mathrm{p}$ values are reported with $\mathrm{p}<0.05$ being accepted as statistically significant.

\section{Results}

Eighty one patients were studied, 76 women and five men. The mean (SD) age and the mean (SD) disease duration at study were 43.4 (12.5) years and 12.7 (8.0) years respectively. Fifty eight $(72 \%)$ were white, seven $(9 \%)$ were
Table 2 Correlation between the Fatigue Severity Scale (FSS) and health status in the eight domains of the SF-36 ( $r$ values and $95 \%$ CI)

\begin{tabular}{ll}
\hline Domain & Correlation with FSS \\
\hline Physical function & $-0.66(-0.79 \text { to }-0.46)^{\star}$ \\
Role physical & $-0.58(-0.74 \text { to }-0.37)^{\star}$ \\
General health & $-0.82(-0.90 \text { to } 0.70)^{\star}$ \\
Bodily pain & $-0.55(-0.71 \text { to }-0.32)^{\star}$ \\
Vitality & $-0.71(-0.83 \text { to }-0.54)^{\star}$ \\
Social function & $-0.57(-0.73 \text { to }-0.31)^{\star}$ \\
Role emotional & $-0.51(-0.69 \text { to }-0.28)^{\star}$ \\
Mental health & $-0.50(-0.68$ to -0.26$) \dagger$ \\
\hline
\end{tabular}

${ }^{\star} \mathrm{p}=0.0001, \mathrm{tp}=0.0002$.

black, and 16 (19\%) were other races. Twenty four $(30 \%)$ had a university education, 28 $(35 \%)$ had college education, 25 (31\%) completed high school, and four (5\%) had elementary or less education. The 81 patients had complete information for the SLEDAI, SLAM-R, SLICC, FSS, and tender point scores. The SF-36 was completed in 51 patients. There were no differences with regard to patient characteristics, disease activity, damage, fatigue severity or tender point score between the 51 with complete information and the 30 who did not complete the SF-36 (data not shown).

The mean SLEDAI and SLAM-R scores at study were low (table 1 ). The level of fatigue reported was high; mean (SD) 6.08 (2.7). A previous study of healthy adults ${ }^{10}$ reported a mean score of $2.3(0.7)$. Table 1 also displays the scores for each domain of the SF-36 in SLE patients that were lower than those reported for healthy US women. ${ }^{12}$ There was a good correlation between the SLEDAI and SLAM-R scores $(r=0.44(0.22,0.58), \mathrm{p}<0.001)$. There was no correlation between the SLEDAI and SLICC damage index $(r=0.03(-0.18,0.25)$ and a poor correlation between the SLAM-R and the SLICC $(r=0.27(0.06,0.47), p=0.01)$.

The fatigue severity score had a poor correlation with disease activity by both indices: SLAM-R $\quad(r=0.26, \quad(0.04,0.45) \quad \mathrm{p}=0.02)$, SLEDAI $(r=0.22 \quad(-0.01,0.41), \mathrm{p}=0.05)$, SLAM-R with fatigue score removed $(r=0.11$ $(-0.12,0.32), p=N S)$. There was also no relation between the FSS and SLICC $(r=0.20$ $(-0.02,0.40), \mathrm{p}=0.07)$. The FSS correlated well with the tender point count $(r=0.46$, $(0.27,0.62) \mathrm{p}<0.001)$. With regard to QOL, there were strong negative correlations between the FSS and all domains of the SF-36 with correlation coefficients ranging from -0.50 to -0.82 (table 2 ). The negative correlations reflect the fact that a high FSS reflects more fatigue, whereas a high SF-36 reflects better quality of life. The variance in fatigue accounted for by the SLEDAI and SLICC were $4.8 \%$ and $4.0 \%$ respectively. In contrast, the dimensions of the SF-36 accounted for $25-67 \%$ of the variance in the FSS.

\section{Discussion}

We have found that in an outpatient lupus population the severity of fatigue correlates strongly with health status as measured by the SF-36. There was no significant correlation with disease activity, measured by the SLEDAI or SLAM-R. Also, there was no correlation 
between fatigue and the SLICC/ACR damage index. The reporting of fatigue in SLE is therefore more reflective of the health status/QOL dimension of disease than disease activity or damage.

Fatigue severity correlated strongly with all domains of the SF-36. In addition, all the values of the SF-36 in SLE were lower than the reported normal values for a healthy female population. This suggests that there is a relation between poor QOL and the degree of fatigue reported that confirms our previous findings using the MOS SF- $20 .^{6}$ In addition, we have found that fatigue severity correlates with the tender point count and previously we have noted a correlation with higher scores on a depression inventory. ${ }^{6}$ One potential explanation for this observation is that QOL and depression represent self report scales, and tenderness is partly dependent on the patient's own self reporting. In contrast, disease activity and damage scales rely on the objective assessment of a trained physician. Therefore all the "patient centred" scales may be expected to closely correlate with a self reported scale of fatigue. It has been noted in studies of pain in RA that depression correlated well with self reported pain but not with observer rated pain behaviours. ${ }^{14}$ Certain pain behaviours did correlate well with disease activity. ${ }^{14}$ Objective study of fatigue associated behaviours has not been undertaken, but may be necessary to discover if our findings have been confounded by the self report nature of the questionnaires.

The correlation between the SLAM-R and SLEDAI is lower than previously reported. ${ }^{15}$ This initial study was performed by members of an international study group using "paper patients" over a broad range of disease activity. The lower correlation in our study may more closely reflect the correlation to be expected in a general clinic situation within a group of patients with a generally lower range of disease activity. We cannot exclude the possibility that there may be variation in the degree of fatigue that may occur during a major flare of disease. Studying patients over time would permit this question to be investigated and also enable assessment of the level of fatigue as a function of disease activity in individual patients. With regard to the SLAM-R, the modest correlation with fatigue severity was lost when the score for fatigue was omitted. Although each element of the SLAM-R demands attribution to active SLE, ${ }^{7}$ the scoring of fatigue and its attribution to active disease does, by necessity, require a degree of interpretation and clinical judgment. The inclusion of such subjective data in an activity score may therefore increase the risk of rater variability. ${ }^{8}$

A study such as this cannot discover the aetiology of fatigue in SLE. Fatigue may be a cause or consequence of a poor perception of health status. Alternatively, fatigue and perceived QOL/health status may be closely associated with other key psychosocial factors not as- sessed by this study. It is also possible that fatigue may reflect more subtle changes in CNS structure and physiology that cannot be assessed by activity and damage instruments currently used. Tests of neurocognitive function and/or sleep physiology may allow you to further assess CNS function in these patients. They may also provide objective assessment of some fatigue related behaviours that would in part elucidate the potential confounders described earlier.

Therefore, in an outpatient SLE population we have found that self reported fatigue severity has a strong negative correlation with all domains of the SF-36. There was no correlation with disease activity or organ damage. It would seem that in patients with SLE, factors associated with QOL/health status dimension of disease have a greater influence on the severity of fatigue than the degree of disease activity.

Funding: Dr I N Bruce is in receipt of the Geoffrey Carr Lupus Fellowship from the Ontario Lupus Association. This study was also supported in part by a grant from the Arthritis Society of Canada.

1 Schur PH. Clinical features of SLE. Kelley WN, Harris Jr ED, Ruddy S, Sledge CB, eds. Textbook of rheumatology. ED, Ruddy S, Sledge CB, eds. Textbo

2 Liang MH, Rogers M, Larson M, Eaton HM, Murawski BJ, Taylor JE, et al. The psychosocial impact of systemic lupus erythematosus and rheumatoid arthritis. Arthritis Rheum 1984;27:13-19.

3 Liang MH, Socher SA, Larson MG, Schur PH. Reliability and validity of six systems for the clinical assessment of disease activity in systemic lupus erythematosus. Arthritis Rheum 1989;32:1107-18.

4 Middleton GD, McFarlin JE, Lipsky PE. The prevalence and clinical impact of fibromyalgia in systemic lupus erythematosus. Arthritis Rheum 1994;37:1181-8.

5 Gladman DD, Urowitz MB, Gough J, MacKinnon. Fibromyalgia is a major contributor to quality of life in Fibromyalgia is a major contributor

6 Wang B, Gladman DD, Urowitz MB. Fatigue in lupus is not Wang B, Gladman DD, Urowitz MB. Fatigue in lupus is not correlated.$$
\text { 892-5 }
$$

7 Bombardier C, Gladman DD, Urowitz MB, Caron D, Chang $\mathrm{CH}$. Derivation of the SLEDAI. A disease activity index for lupus patients. The Committee on Prognosis Studies in SLE. Arthritis Rheum 1992;35:630-40.

8 Gladman DD, Urowitz MB, Fortin P, Isenberg D, Goldsmith C, Gordon C, et al. Systemic Lupus International Collaborating Clinics Conference on assessment of lupus flare and quality of life measures in SLE. J Rheumatol 1996;23:1953-5.

9 Tan EM, Cohen AS, Fries JF, Masi AT, McShane DJ, Rothfield NF, et al. The 1982 revised criteria for the classfication field NF, et al. The 1982 revised criteria for the classfication of systemic

10 Gladman DD, Urowitz MB, Goldsmith CH, Fortin P, Ginzler E, Gordon C, et al. The reliability of the Systemic Lupus International Collaborating Clinics/American College of Rheumatology Damage Index in patients with systemic lupus erythematosus. Arthritis Rheum 1997;40 809-13.

11 Krupp LB, LaRocca NG, Muir-Nash J, Steinberg AD. The fatigue severity scale. Application to patients with multiple sclerosis and systemic lupus erythematosus. Arch Neurol 1989;46:1121-3.

12 Gladman DD, Urowitz MB, Ong A, Gough J, MacKinnon A. A comparison of five health status instruments in A. A comparison of five health status instruments in patients

13 Ware JE, Snow KK, Kosinski M, Gandek B. SF-36 health survey manual and interpretation guide. Boston: New England Medical Center, 1993.

14 McDaniel LK, Anderson KO, Bradley LA, Young LD, Turner RA, Agudelo CA, et al. Development of an observation method for assessing pain behaviour in rheumatoid arthritis patients. Pain $1986 ; 24: 165-84$

15 Gladman DD, Goldsmith $\mathrm{CH}$, Urowitz $\mathrm{MB}$, Bacon P, Bombardier C, Isenberg D, et al. Sensitivity to change of 3 systemic lupus erythematosus disease activity indices: International validation. J Rheumatol 1994;21:1468-71. 